\title{
Asymptotic Analysis of Correlated Multi-Antenna Broadcast Channels
}

\author{
Romain Couillet \\ ST-NXP Wireless / Supélec \\ Route des Lucioles, 505 \\ 06904 Sophia-Antipolis, France \\ Email: romain.couillet@supelec.fr
}

\author{
Sebastian Wagner \\ ST-NXP Wireless / Eurecom Institute \\ Route des Lucioles, 505 \\ 06904 Sophia-Antipolis, France \\ Email: sebastian.wagner@eurecom.fr
}

\author{
Mérouane Debbah \\ Supélec \\ 3, Rue Joliot-Curie \\ 91192 Gif sur Yvette, France \\ Email: merouane.debbah@supelec.fr
}

\begin{abstract}
In this paper we consider the MIMO broadcast channel with antenna correlation at the transmitter and receiver. We derive the theoretical sum rate of systems with a large number of antennas for zero-forcing and regularized zero-forcing precoders. Particularly, we apply the results to volume-limited devices where the correlation originates from a dense antenna packing. Throughout this contribution we make extensive use of recent tools from random matrix theory. Simulations confirm the theoretical claims and also indicate that in most scenarios the asymptotic derivations applied to a finite number of users give good approximations of the true ergodic sum rate.
\end{abstract}

\section{INTRODUCTION}

The multiple-input multiple-output (MIMO) broadcast channel (BC) has the potential to significantly increase the system capacity by exploiting the spatial domain to communicate to several users in the same time-frequency resource. Recently, it has been shown that the capacity region of the MIMO-BC is achieved by dirty-paper coding [5]. However, for both practical and analytical reasons, it is convenient to use linear precoding techniques such as zero-forcing (ZF) or regularized-ZF (R-ZF) precoding. The asymptotic achievable sum rate of $\mathrm{ZF}$ and $\mathrm{R}-\mathrm{ZF}$ for a large number of users and under Gaussian i.i.d. channels has been studied in [13] and [14]. In this paper we extend those analysis by incorporating correlation between antennas following the Kronecker channel model. Our approach is based on recent results of random matrix theory (RMT) and particularly on the spectral analysis of large random matrices. A similar approach has been pursued for the point-to-point MIMO channel in [6] and [19]. As an application we study the effect of dense antenna packing on the throughput which has already been studied for the pointto-point MIMO channel cf. [3], [16] and [18].

The remainder of this paper is organized as follows: Section II briefly reviews various tools of random matrix theory. In Section III, the system model of the correlated MIMO broadcast channel is introduced. In Sections IV and V, the asymptotic sum rates of the correlated MIMO broadcast channel with ZF and R-ZF precoding are derived. Section VI provides simulation results which corroborate the theoretical derivations. Finally in Section VII we provide our conclusions.

Notation: In the following, boldface lower-case symbols represent vectors, capital boldface characters denote matrices ( $\mathbf{I}_{N}$ is the $N \times N$ identity matrix). The Hermitian transpose is denoted $(\cdot)^{\mathrm{H}}$. The operator $\operatorname{tr} \mathbf{X}$ represents the trace of matrix $\mathbf{X}$. The eigenvalue distribution of an Hermitian random matrix $\mathbf{X}$ is $\mu_{\mathbf{X}}(x)$. The symbol $\mathrm{E}[\cdot]$ denotes expectation. The derivative of a function $f$ of a single variable $x$ is denoted $\frac{\mathrm{d}}{\mathrm{d} x} f$.

\section{RANDOM MATRIX THEORY TOOLS}

Since the pioneering work of Wigner [12] on the asymptotic eigenvalue distribution of random Hermitian matrices, random matrix theory has grown into a new field of research in theoretical physics and applied probability. The main application to the telecommunication realm lies in the derivation of asymptotic results for large matrices. Specifically, the eigenvalue distribution of large Hermitian matrices converges, in many practical cases, to a definite probability distribution, called the empirical distribution of the random matrix. In several occasions in this work, we need to compute the trace of the resolvent $(\mathbf{X}-z \mathbf{I})^{-1}$ of random Hermitian matrices $\mathbf{X}$, which is given by the Stieltjes transform $\mathcal{S}_{\mathbf{X}}$,

$$
\mathcal{S}_{\mathbf{X}}(z)=\int_{-\infty}^{+\infty} \frac{1}{x-z} \mu_{\mathbf{X}}(x) \mathrm{d} x
$$

where $\mu_{\mathbf{X}}(x)$ is the empirical distribution of $\mathbf{X}$.

Silverstein [20] derived a fixed-point expression of the Stieltjes transform for i.i.d. random matrices with left- and right-sided correlations in the following theorem,

Theorem 1: Let the entries of the $N \times K$ matrix $\mathbf{W}$ be i.i.d. with zero mean and variance $1 / K$. Let $\mathbf{X}$ and $\mathbf{Q}$ be $N \times N$ Hermitian deterministic matrices with empirical eigenvalue distribution functions converging weakly to $\mu_{\mathbf{X}}$ and $\mu_{\mathbf{Q}}$ almost surely. Let $\mathbf{Y}$ be an $N \times N$ Hermitian matrix with the same eigenvectors as $\mathbf{X}$ and let $f$ be some function mapping the eigenvalues of $\mathbf{X}$ to those of $\mathbf{Y}$. Then, as $K, N \rightarrow \infty$ with $N / K \rightarrow \alpha$ fixed, the Stieltjes transform $\mathcal{S}_{\mathbf{H}}(z)$ of $\mathbf{H}=\mathbf{X}^{1 / 2} \mathbf{W Q W}^{\mathrm{H}} \mathbf{X}^{1 / 2}+\mathbf{Y}$ converges to

$$
\mathcal{S}_{\mathbf{H}}(z)=\int\left(f(x)+x \int \frac{q \cdot \mu_{\mathbf{Q}}(q) \mathrm{d} q}{1+\alpha q \mathcal{T}_{\mathbf{H}}(z)}-z\right)^{-1} \mu_{\mathbf{X}}(x) \mathrm{d} x
$$

where $\mathcal{T}_{\mathbf{H}}$ is the unique solution of the fixed-point equation

$$
\mathcal{T}_{\mathbf{H}}(z)=\int x\left(f(x)+x \int \frac{q \cdot \mu_{\mathbf{Q}}(q) \mathrm{d} q}{1+\alpha q \mathcal{T}_{\mathbf{H}}(z)}-z\right)^{-1} \mu_{\mathbf{X}}(x) \mathrm{d} x
$$


Corollary 2: [8] Let the entries of the $N \times K$ matrix $\mathbf{W}$ be i.i.d. with zero mean and variance $1 / K$. Let $\mathbf{Y}$ be an $N \times N$ Hermitian random matrix with an empirical eigenvalue distribution function converging weakly to $\mu_{\mathbf{Y}}(x)$ almost surely. Moreover, let $\mathbf{Q}$ be a $K \times K$ real diagonal random matrix with an empirical distribution function converging almost surely in distribution to a probability distribution function $\mu_{\mathbf{Q}}(x)$ as $K \rightarrow \infty$. Then almost surely, the empirical eigenvalue distribution of the random matrix:

$$
\mathbf{H}=\mathbf{W Q W}^{\mathrm{H}}+\mathbf{Y}
$$

converges weakly, as $K, N \rightarrow \infty$ but $N / K \rightarrow \alpha$ fixed, to the unique distribution function whose Stieltjes transform satisfies:

$$
\mathcal{S}_{\mathbf{H}}(z)=\mathcal{S}_{\mathbf{Y}}\left(z-\int \frac{q}{1+\alpha q \mathcal{S}_{\mathbf{H}}(z)} \mu_{\mathbf{Q}}(q) \mathrm{d} q\right)
$$

\section{System MODEL}

Consider the scenario where one transmitter with $M$ antennas communicates to $K$ single-antenna receivers. In addition we assume that $M / K \geq 1$. Under this assumptions the received signal vector $\mathbf{y} \in \mathbb{C}^{K}$ of a narrow-band communication system reads

$$
\mathbf{y}=\mathbf{H x}+\mathbf{n}
$$

with transmit vector $\mathbf{x} \in \mathbb{C}^{M}$, channel matrix $\mathbf{H} \in \mathbb{C}^{K \times M}$ and noise vector $\mathbf{n} \in \mathcal{C N}\left(0, \sigma^{2} \mathbf{I}_{K}\right)$. Furthermore the channel vector $\mathbf{h}_{k}^{\mathrm{H}}$ of user $k$ is the $k$ th row of $\mathbf{H}$. The transmit signal vector $\mathbf{x}$ is obtained from the symbol vector $\mathbf{s} \in \mathbb{C}^{K}$ by a linear precoding $\mathbf{G} \in \mathbb{C}^{M \times K}$

$$
\mathrm{x}=\mathrm{Gs}
$$

where $\mathrm{E}\left[\mathbf{s s}^{\mathrm{H}}\right]=\mathbf{I}_{K}$ and $\mathbf{G}$ satisfies the total transmit power constraint

$$
\mathrm{E}\left[\mathbf{x}^{\mathrm{H}} \mathbf{x}\right]=\mathrm{E}\left[\mathbf{s}^{\mathrm{H}} \mathbf{G}^{\mathrm{H}} \mathbf{G} \mathbf{s}\right]=1
$$

The symbol received by user $k$ is given by

$$
y_{k}=\mathbf{h}_{k}^{\mathrm{H}} \mathbf{G} \mathbf{h}_{k} s_{k}+\sum_{i=1, i \neq k}^{K} \mathbf{h}_{k}^{\mathrm{H}} \mathbf{G} \mathbf{h}_{i} s_{i}+n_{k}
$$

We further assume that the channel $\mathbf{H}$ follows the widely used [6], [19] Kronecker model.

$$
\mathbf{H}=\boldsymbol{\Theta}_{\mathrm{R}}^{1 / 2} \mathbf{H}_{w} \Theta_{\mathrm{T}}^{1 / 2}
$$

where $\mathbf{H}_{w} \in \mathbb{C}^{K \times M}$ is a matrix with standard i.i.d. Gaussian (white) entries. The deterministic correlation matrices $\Theta_{\mathrm{T}}^{1 / 2} \in$ $\mathbb{C}^{M \times M}$ and $\Theta_{\mathrm{R}}^{1 / 2} \in \mathbb{C}^{K \times K}$ at the transmitter and receiver, respectively, are Hermitian positive-definite. In the MIMO broadcast channel the distance between the users is supposed to be sufficiently large compared to the signal wavelength $\lambda$, i.e. $\Theta_{\mathrm{R}}^{1 / 2}=\mathbf{I}_{K}$.

A widely used correlation model, which we will consider as a case study in the simulation Section VI, is based on Jakes' model [7], i.e. $\left(\boldsymbol{\Theta}_{\mathrm{R}}\right)_{i j}=\mathrm{J}_{0}\left(2 \pi d_{i j} / \lambda\right)$, where $\mathrm{J}_{0}$ is the zeroorder Bessel function of the first kind and $d_{i j}$ is the distance between transmit antennas $i$ and $j$. In the particular case of a

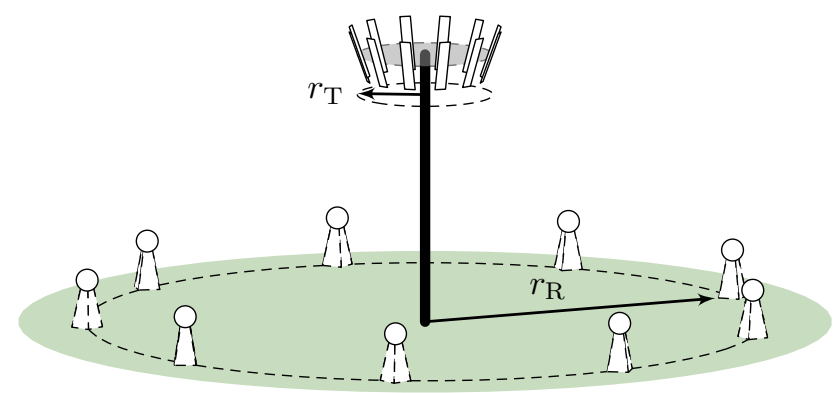

Fig. 1. Broadcast channel with uniform circular antenna arrays at the transmitter and the receiver

uniform circular array (UCA) of radius $r_{\mathrm{T}}$ at the transmitter the eigenvalues of $\Theta_{\mathrm{T}}$ are directly given by [3]

$$
\mu_{\boldsymbol{\Theta}_{\mathrm{T}}}(\lambda)=\lim _{M \rightarrow \infty} \sum_{m=1}^{M} \delta\left(\lambda-\mathrm{J}_{m}^{2}\left(2 \pi r_{\mathrm{T}} / \lambda\right)\right)
$$

It is shown by Pollock in [3] that for $m>\left\lceil\pi e r_{\mathrm{T}} / \lambda\right\rceil$ the eigenvalues $\mathrm{J}_{m}^{2}\left(2 \pi r_{\mathrm{T}} / \lambda\right)$ are very small. Therefore, Pollock refers to $\left[\pi e r_{\mathrm{T}} / \lambda\right]$ as the number of degrees of freedom provided by the communication channel. The UCA model is depicted in Figure 1.

In the subsequent sections we derive asymptotic sum rates for large $(K, M)$ such that $M / K \rightarrow \beta \geq 1$ in case of linear precoding at the transmitter and equal power allocation across the users' symbols $\mathbf{s}^{1}$

\section{ZERO-FORCING PRECODING}

The zero-forcing (ZF) precoding annihilates all the interuser interference by performing an inversion of the channel matrix $\mathbf{H}$ at the transmitter. The asymptotic behavior of the channel inversion precoding scheme with uncorrelated antennas has been studied in [13], [14]. The precoding matrix is given by

$$
\mathbf{G}_{\mathrm{zf}}= \begin{cases}\alpha_{M} \mathbf{H}^{-1} & , \text { if } M / K=1 \\ \alpha_{M} \mathbf{H}^{\mathrm{H}}\left(\mathbf{H H}^{\mathrm{H}}\right)^{-1} & , \text { if } M / K>1\end{cases}
$$

where the parameter $\alpha_{M}$ is set to fulfill the transmission power constraint (8). Thus, we have

$$
\alpha_{M}^{2}=\frac{1}{\operatorname{tr}\left(\mathbf{H} \mathbf{H}^{\mathrm{H}}\right)^{-1}}
$$

and the system model in (6) becomes

$$
\mathbf{y}=\alpha_{M} \mathbf{s}+\mathbf{n}
$$

The signal-to-interference plus noise ratio (SINR) $\gamma_{k, M}$ for user $k$ is defined as

$$
\gamma_{k, M}=\frac{\mathrm{E}\left[x_{k}^{*} x_{k}\right]}{\sigma^{2}}=\frac{\alpha_{M}^{2}}{\sigma^{2}}
$$

which is therefore independent of the selected user $k$.

\footnotetext{
${ }^{1}$ note that the aforementioned sum rate with equal power allocation does not necessarily correspond to the achievable sum rate under linear precoding.
} 
Let us denote $\mathbf{H}^{\prime}=\frac{1}{\sqrt{M}} \mathbf{H}$ and $\mathbf{H}_{w}^{\prime}=\frac{1}{\sqrt{M}} \mathbf{H}_{w}$. It follows from (13)

$$
\alpha_{M}^{2}=\frac{1}{\frac{1}{M} \operatorname{tr}\left(\mathbf{H}^{\prime} \mathbf{H}^{\prime \mathbf{H}}\right)^{-1}}
$$

In the limit $M \rightarrow \infty$ with $M / K \rightarrow \beta$, the denominator of Equation (16) verifies

$$
\frac{1}{M} \operatorname{tr}\left(\mathbf{H}^{\prime} \mathbf{H}^{\prime \mathbf{H}}\right)^{-1} \rightarrow \frac{1}{\beta} \int \frac{1}{\lambda} \mu_{\mathbf{H}^{\prime} \mathbf{H}^{\prime \mathbf{H}}}(\lambda) \mathrm{d} \lambda=\frac{1}{\beta} \mathcal{S}_{\mathbf{H}^{\prime} \mathbf{H}^{\prime \mathbf{H}}}(0)
$$

According to Corollary $2, \mathcal{S}_{\mathbf{H}^{\prime} \mathbf{H}^{\prime \mathbf{H}}}(0)$ is the solution of

$$
\mathcal{S}_{\mathbf{H}^{\prime} \mathbf{H}^{\prime \mathbf{H}}}(0)=\left(\int \frac{\lambda}{1+\frac{1}{\beta} \lambda \mathcal{S}_{\mathbf{H}^{\prime} \mathbf{H}^{\prime \mathbf{H}}}(0)} \mu_{\boldsymbol{\Theta}_{\mathrm{T}}}(\lambda) \mathrm{d} \lambda\right)^{-1}
$$

As a consequence, for large $(K, M)$

$$
\gamma_{k . M}=\frac{\alpha_{M}^{2}}{\sigma^{2}} \rightarrow \frac{\beta}{\sigma^{2} \mathcal{S}_{\mathbf{H}^{\prime} \mathbf{H}^{\prime \mathbf{H}}}(0)}
$$

and the rate of user $k$ is

$$
\begin{aligned}
\mathcal{R}_{\mathrm{zf}, k} & =\log \left(1+\gamma_{k . M}\right) \\
& \rightarrow \log \left(1+\frac{\beta}{\sigma^{2} \mathcal{S}_{\mathbf{H}^{\prime} \mathbf{H}^{\prime \mathbf{H}}}(0)}\right)
\end{aligned}
$$

which is independent of $k$.

Remark 1: The previous results can be generalized by allowing each user to be equipped with $N$ correlated antennas. In the channel model $\mathbf{y}=\mathbf{H} \mathbf{x}+\mathbf{n}$, the transmit/receive and noise vectors of the $K$ users are stacked in vectors of size $K N$. We have the following correlation for the concatenated channel $\mathbf{H}$

$$
\mathbf{H}=\boldsymbol{\Theta}_{\mathrm{R}}^{1 / 2} \mathbf{H}_{w} \boldsymbol{\Theta}_{\mathrm{T}}^{1 / 2}
$$

where $\Theta_{\mathrm{R}}$ is a $K N \times K N$ block-diagonal matrix and $\boldsymbol{\Theta}_{\mathrm{T}}$ is the classical $M \times M$ transmit correlation matrix.

With the same derivation as previously we obtain

$$
\mathcal{R}_{\mathrm{zf}, k} \rightarrow \log \left(1+\frac{\beta}{\sigma^{2} \mathcal{S}_{\mathbf{H}^{\prime} \mathbf{H}^{\prime \mathbf{H}}}(0)}\right)
$$

where $\mathcal{S}_{\mathbf{H}^{\prime} \mathbf{H}^{\prime \mathbf{H}}}(0)$ is now obtained from the more general Theorem 1

$$
\mathcal{S}_{\mathbf{H}^{\prime} \mathbf{H}^{\prime}}(0)=\mathcal{S}_{\Theta_{\mathrm{R}}}(0) \cdot\left(\int \frac{\lambda}{1+\frac{\lambda}{\beta} \frac{\mathcal{S}_{\mathrm{H}^{\prime} \mathbf{H}^{\prime}}(0)}{\mathcal{S}_{\Theta_{\mathrm{R}}}(0)}} \mu_{\boldsymbol{\Theta}_{\mathrm{R}}}(\lambda) \mathrm{d} \lambda\right)^{-1}
$$

where $\beta=\lim _{M \rightarrow \infty} \frac{M}{K N}$. The complete demonstration of this result is provided in [20].

\section{Regularized Zero-Forcing Precoding}

Let us now consider R-ZF precoding, for which the precoding matrix is given by

$$
\begin{aligned}
\mathbf{G}_{\mathrm{rzf}} & =\mathbf{H}^{\mathrm{H}}\left(\mathbf{H} \mathbf{H}^{\mathrm{H}}+M \alpha \mathbf{I}_{K}\right)^{-1} \\
& \stackrel{(a)}{=}\left(\mathbf{H}^{\mathrm{H}} \mathbf{H}+M \alpha \mathbf{I}_{M}\right)^{-1} \mathbf{H}^{\mathbf{H}}
\end{aligned}
$$

where $(a)$ follows from the matrix inversion lemma (MIL) and we use $\alpha$ to satisfy the transmit power constraint in (8). The regularization term $M \alpha \mathbf{I}_{K}$ contains the factor $M$ to ensure that, as $(K, M)$ grow large, both $\operatorname{tr}\left(\mathbf{H} \mathbf{H}^{\mathrm{H}}\right)$ and $\operatorname{tr}\left(M \alpha \mathbf{I}_{K}\right)$ grow with the same order of magnitude. For large $(K, M)$, the constraint (8) determines $\alpha$ as follows

$$
\begin{aligned}
1 & =\frac{1}{M} \operatorname{tr}\left[\mathbf{H}^{\prime}{ }^{\mathrm{H}} \mathbf{H}^{\prime}\left(\mathbf{H}^{\prime}{ }^{\mathrm{H}} \mathbf{H}^{\prime}+\alpha \mathbf{I}_{M}\right)^{-2}\right] \\
& \rightarrow \int \frac{\nu}{(\nu+\alpha)^{2}} \mu_{\mathbf{H}^{\prime \mathrm{H}} \mathbf{H}^{\prime}}(\nu) \mathrm{d} \nu \\
& =\int\left(\frac{1}{(\nu+\alpha)}-\frac{\alpha}{(\nu+\alpha)^{2}}\right) \mu_{\mathbf{H}^{\prime}{ }^{\mathrm{H}} \mathbf{H}^{\prime}}(\nu) \mathrm{d} \nu \\
& =\mathcal{S}_{\mathbf{H}^{\prime}{ }^{\mathrm{H}} \mathbf{H}^{\prime}}(-\alpha)-\alpha \frac{\mathrm{d}}{\mathrm{d} x} \mathcal{S}_{\mathbf{H}^{\prime \mathrm{H}} \mathbf{H}^{\prime}}(-\alpha)
\end{aligned}
$$

with, from classical results on Stieltjes transforms [9],

$$
\begin{aligned}
\mathcal{S}_{\mathbf{H}^{\prime \prime}{ }^{H} \mathbf{H}^{\prime}}(z) & =\mathcal{S}_{\boldsymbol{\Theta}_{\mathrm{T}}^{1 / 2} \mathbf{H}_{w}^{\prime}{ }^{\mathrm{H}} \mathbf{H}_{w}^{\prime} \boldsymbol{\Theta}_{\mathrm{T}}^{1 / 2}(z)} \\
& =\frac{1}{\beta} \mathcal{S}_{\mathbf{H}_{w}^{\prime} \boldsymbol{\Theta}_{\mathrm{T}} \mathbf{H}_{w}^{\prime}{ }^{\mathrm{H}}}(z)-\frac{\beta-1}{\beta z}
\end{aligned}
$$

where $\mathcal{S}_{\mathbf{H}_{w}^{\prime} \boldsymbol{\Theta}_{\mathrm{T}} \mathbf{H}_{w}^{\prime}}(z)$ satisfies the fixed-point equation (2)

$$
\mathcal{S}_{\mathbf{H}_{w}^{\prime} \boldsymbol{\Theta}_{\mathrm{T}} \mathbf{H}_{w}^{\prime}{ }^{\mathrm{H}}}(z)=\left(\int \frac{\lambda \mu_{\boldsymbol{\Theta}_{\mathrm{T}}}(\lambda) \mathrm{d} \lambda}{1+\frac{\lambda}{\beta} \mathcal{S}_{\mathbf{H}_{w}^{\prime} \boldsymbol{\Theta}_{\mathrm{T}} \mathbf{H}_{w}^{\prime}{ }^{\mathrm{H}}}(z)}-z\right)^{-1}
$$

and, from (33),

$$
\frac{\mathrm{d}}{\mathrm{d} x} \mathcal{S}_{\Theta_{\mathrm{T}}^{1 / 2} \mathbf{H}_{w}^{\prime}{ }^{\mathrm{H}} \mathbf{H}_{w}^{\prime} \Theta_{\mathrm{T}}^{1 / 2}}(z)=\frac{1}{\beta} \frac{\mathrm{d}}{\mathrm{d} x} \mathcal{S}_{\mathbf{H}_{w}^{\prime} \boldsymbol{\Theta}_{\mathrm{T}} \mathbf{H}_{w}^{\prime}{ }^{\mathrm{H}}}(z)+\frac{\beta-1}{\beta z^{2}}
$$

where after differentiation of (34)

$$
\frac{\mathrm{d}}{\mathrm{d} x} \mathcal{S}_{\mathbf{H}_{w}^{\prime} \boldsymbol{\Theta}_{\mathrm{T}} \mathbf{H}_{w}^{\prime}{ }^{\mathrm{H}}}(z)=\frac{1+\int \frac{\frac{\lambda^{2}}{\beta} \frac{\mathrm{d}}{\mathrm{d} x} \mathcal{S}_{\mathbf{H}_{w}^{\prime} \boldsymbol{\Theta}_{\mathrm{T}} \mathbf{H}_{w}^{\prime}{ }^{\mathrm{H}}}(z)}{\left(1+\frac{\lambda}{\beta} \mathcal{S}_{\mathbf{H}_{w}^{\prime} \boldsymbol{\Theta}_{\mathrm{T}} \mathbf{H}_{w}^{\prime}{ }^{\mathrm{H}}}(z)\right)^{2}} \mu_{\boldsymbol{\Theta}_{\mathrm{T}}}(\lambda) \mathrm{d} \lambda}{\left(\mathcal{S}_{\mathbf{H}_{w}^{\prime} \boldsymbol{\Theta}_{\mathrm{T}} \mathbf{H}_{w}^{\prime}{ }^{\mathrm{H}}}(z)\right)^{2}}
$$

After determining $\alpha$, we derive the asymptotic SINR per user. The received signal is given by

$$
\mathbf{y}=\mathbf{H}^{\prime}\left(\mathbf{H}^{\prime \mathrm{H}^{\mathrm{H}}} \mathbf{H}^{\prime}+\alpha \mathbf{I}_{M}\right)^{-1} \mathbf{H}^{\prime \mathrm{H}} \mathbf{s}+\mathbf{n}
$$

Denote $\mathbf{H}^{\prime \mathbf{H}}=\left[\mathbf{h}_{1}^{\prime}, \ldots, \mathbf{h}_{K}^{\prime}\right]$ and $\mathbf{W}=\left(\mathbf{H}^{\prime}{ }^{\mathrm{H}} \mathbf{H}^{\prime}+\alpha \mathbf{I}_{M}\right)^{-1}$. We will focus on user $k$ without loss of generality. The received symbol of user $k$ is

$$
y_{k}=\mathbf{h}_{k}^{\prime}{ }^{\mathrm{H}} \mathbf{W} \mathbf{h}_{k}^{\prime} s_{k}+\sum_{i=1, i \neq k}^{K} \mathbf{h}_{k}^{\prime}{ }^{\mathrm{H}} \mathbf{W} \mathbf{h}_{i}^{\prime} s_{i}+n_{k}
$$

and the SINR of user $k$ reads

$$
\gamma_{k, M}=\frac{\left|\mathbf{h}_{k}^{\prime}{ }^{\mathrm{H}} \mathbf{W} \mathbf{h}_{k}^{\prime}\right|^{2}}{\mathbf{h}_{k}^{\prime}{ }^{\mathrm{H}} \mathbf{W} \mathbf{U}_{k}^{\mathrm{H}} \mathbf{U}_{k} \mathbf{W} \mathbf{h}_{k}^{\prime}+\sigma^{2}}
$$

where we denote $\mathbf{U}_{k}^{\mathrm{H}}=\left[\mathbf{h}_{1}^{\prime}, \ldots, \mathbf{h}_{k-1}^{\prime}, \mathbf{h}_{k+1}^{\prime}, \ldots, \mathbf{h}_{K}^{\prime}\right]$ (in other words, we remove column/user $k$ ). In the subsequent derivation of the asymptotic SINR of user $k$ we need the following result from RMT.

Lemma 3: [10] Let $\mathbf{A}$ be a deterministic $N \times N$ complex matrix with uniformly bounded spectral radius for all $N$. Let 


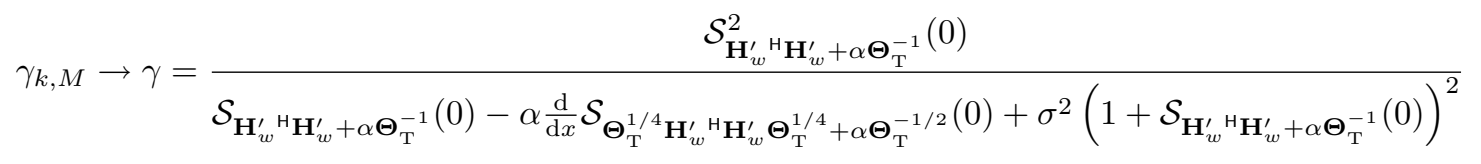

$\mathbf{x}=\frac{1}{\sqrt{N}}\left[x_{1}, \ldots, x_{N}\right]^{\top}$ where the $\left\{x_{i}\right\}$ are i.i.d. complex random variables with zero mean, unit variance and finite eighth moment. Then

$$
\mathrm{E}\left[\left|\mathbf{x}^{\mathrm{H}} \mathbf{A} \mathbf{x}-\frac{1}{N} \operatorname{tr} \mathbf{A}\right|^{4}\right] \leq \frac{c}{N^{2}}
$$

where $c$ is a constant that does not depend on $N$ or $\mathbf{A}$.

Corollary 4: With the hypothesis of Lemma 3,

$$
\mathbf{x}^{\mathrm{H}} \mathbf{A} \mathbf{x}-\frac{1}{N} \operatorname{tr} \mathbf{A} \rightarrow 0
$$

almost surely.

Applying the MIL on the matrix $\mathbf{W}$ yields

$$
\mathbf{h}_{k}^{\prime}{ }^{\mathrm{H}} \mathbf{W}=\frac{\mathbf{h}_{k}^{\prime \mathrm{H}}\left(\mathbf{U}_{k}^{\mathrm{H}} \mathbf{U}_{k}+\alpha \mathbf{I}_{M}\right)^{-1}}{1+\mathbf{h}_{k}^{\prime}{ }^{\mathrm{H}}\left(\mathbf{U}_{k}^{\mathrm{H}} \mathbf{U}_{k}+\alpha \mathbf{I}_{M}\right)^{-1} \mathbf{h}_{k}^{\prime}}
$$

We apply the following change of variables: $\mathbf{h}_{k}^{\prime}=\Theta_{\mathrm{T}}^{1 / 2} \mathbf{k}_{k}^{\prime}$ and $\mathbf{U}_{k}=\mathbf{V}_{k} \Theta_{\mathrm{T}}^{1 / 2}$ so that $\mathbf{k}_{k}^{\prime}$ is independent of $\mathbf{V}_{k}$ and both multidimensional variables have i.i.d. entries. Thus, we can use Corollary 4

$$
\begin{aligned}
\mathbf{h}_{k}^{\prime}{ }^{\mathrm{H}} \mathbf{W} \mathbf{h}_{k}^{\prime} & =\frac{\mathbf{k}_{k}^{\prime}{ }^{\mathrm{H}}\left(\mathbf{V}_{k}^{\mathrm{H}} \mathbf{V}_{k}+\alpha \mathbf{\Theta}_{\mathrm{T}}^{-1}\right)^{-1} \mathbf{k}_{k}^{\prime}}{1+\mathbf{k}_{k}^{\prime \mathrm{H}}\left(\mathbf{V}_{k}^{\mathrm{H}} \mathbf{V}_{k}+\alpha \boldsymbol{\Theta}_{\mathrm{T}}^{-1}\right)^{-1} \mathbf{k}_{k}^{\prime}} \\
& \rightarrow \lim _{M \rightarrow \infty} \frac{\frac{1}{M} \operatorname{tr}\left[\left(\mathbf{V}_{k}^{\mathrm{H}} \mathbf{V}_{k}+\alpha \mathbf{\Theta}_{\mathrm{T}}^{-1}\right)^{-1}\right]}{1+\frac{1}{M} \operatorname{tr}\left[\left(\mathbf{V}_{k}^{\mathrm{H}} \mathbf{V}_{k}+\alpha \boldsymbol{\Theta}_{\mathrm{T}}^{-1}\right)^{-1}\right]} \\
& =\frac{\mathcal{S}_{\mathbf{V}_{k}^{\mathrm{H}} \mathbf{V}_{k}+\alpha \boldsymbol{\Theta}_{\mathrm{T}}^{-1}}(0)}{1+\mathcal{S}_{\mathbf{V}_{k}^{\mathrm{H}} \mathbf{V}_{k}+\alpha \boldsymbol{\Theta}_{\mathrm{T}}^{-1}}(0)}
\end{aligned}
$$

Similarly, applying twice the MIL to the denominator of (39), we have

$$
\begin{aligned}
& \mathbf{h}_{k}^{\prime}{ }^{\mathrm{H}} \mathbf{W} \mathbf{U}_{k}^{\mathrm{H}} \mathbf{U}_{k} \mathbf{W} \mathbf{h}_{k}^{\prime} \\
& =\frac{\mathbf{h}_{k}^{\prime}{ }^{\mathrm{H}}\left(\mathbf{U}_{k}^{\mathrm{H}} \mathbf{U}_{k}+\alpha \mathbf{I}_{M}\right)^{-1} \mathbf{U}_{k}^{\mathrm{H}} \mathbf{U}_{k}\left(\mathbf{U}_{k}^{\mathrm{H}} \mathbf{U}_{k}+\alpha \mathbf{I}_{M}\right)^{-1} \mathbf{h}_{k}^{\prime}}{\left(1+\mathbf{h}_{k}^{\prime}{ }^{\mathrm{H}}\left(\mathbf{U}_{k}^{\mathrm{H}} \mathbf{U}_{k}+\alpha \mathbf{I}_{M}\right)^{-1} \mathbf{h}_{k}^{\prime}\right)^{2}}
\end{aligned}
$$

$$
=\frac{\mathbf{k}_{k}^{\prime}{ }^{\mathrm{H}}\left(\mathbf{V}_{k}^{\mathrm{H}} \mathbf{V}_{k}+\alpha \boldsymbol{\Theta}_{\mathrm{T}}^{-1}\right)^{-1} \mathbf{V}_{k}^{\mathrm{H}} \mathbf{V}_{k}\left(\mathbf{V}_{k}^{\mathrm{H}} \mathbf{V}_{k}+\alpha \boldsymbol{\Theta}_{\mathrm{T}}^{-1}\right)^{-1} \mathbf{k}_{k}^{\prime}}{\left(1+\mathbf{k}_{k}^{\prime}{ }^{\mathrm{H}}\left(\mathbf{V}_{k}^{\mathrm{H}} \mathbf{V}_{k}+\alpha \boldsymbol{\Theta}_{\mathrm{T}}^{-1}\right)^{-1} \mathbf{k}_{k}^{\prime}\right)^{2}}
$$$$
\rightarrow \lim _{M \rightarrow \infty} \frac{\frac{1}{M} \operatorname{tr}\left[\left(\mathbf{V}_{k}^{\mathrm{H}} \mathbf{V}_{k}+\alpha \boldsymbol{\Theta}_{\mathrm{T}}^{-1}\right)^{-2} \mathbf{V}_{k}^{\mathrm{H}} \mathbf{V}_{k}\right]}{\left(1+\frac{1}{M} \operatorname{tr}\left[\left(\mathbf{V}_{k}^{\mathrm{H}} \mathbf{V}_{k}+\alpha \boldsymbol{\Theta}_{\mathrm{T}}^{-1}\right)^{-1}\right]\right)^{2}}
$$

where the matrix in the numerator of (49) can be expanded as

$$
\begin{aligned}
& \left(\mathbf{V}_{k}^{\mathrm{H}} \mathbf{V}_{k}+\alpha \boldsymbol{\Theta}_{\mathrm{T}}^{-1}\right)^{-2} \mathbf{V}_{k}^{\mathrm{H}} \mathbf{V}_{k} \\
& =\left(\mathbf{V}_{k}^{\mathrm{H}} \mathbf{V}_{k}+\alpha \boldsymbol{\Theta}_{\mathrm{T}}^{-1}\right)^{-2}\left(\mathbf{V}_{k}^{\mathrm{H}} \mathbf{V}_{k}+\alpha \boldsymbol{\Theta}_{\mathrm{T}}^{-1}-\alpha \boldsymbol{\Theta}_{\mathrm{T}}^{-1}\right) \\
& =\left(\mathbf{V}_{k}^{\mathrm{H}} \mathbf{V}_{k}+\alpha \boldsymbol{\Theta}_{\mathrm{T}}^{-1}\right)^{-1}-\alpha\left(\mathbf{V}_{k}^{\mathrm{H}} \mathbf{V}_{k}+\alpha \boldsymbol{\Theta}_{\mathrm{T}}^{-1}\right)^{-2} \boldsymbol{\Theta}_{\mathrm{T}}^{-1}
\end{aligned}
$$

For the trace of the second term of (51) we obtain

$$
\begin{aligned}
& \operatorname{tr}\left[-\alpha\left(\mathbf{V}_{k}^{\mathrm{H}} \mathbf{V}_{k}+\alpha \boldsymbol{\Theta}_{\mathrm{T}}^{-1}\right)^{-2} \boldsymbol{\Theta}_{\mathrm{T}}^{-1}\right] \\
& =-\alpha \cdot \operatorname{tr}\left[\left(\boldsymbol{\Theta}_{\mathrm{T}}^{1 / 4} \mathbf{V}_{k}^{\mathrm{H}} \mathbf{V}_{k} \boldsymbol{\Theta}_{\mathrm{T}}^{1 / 4}+\alpha \boldsymbol{\Theta}_{\mathrm{T}}^{-1 / 2}\right)^{-2}\right]
\end{aligned}
$$

In the limit $M \rightarrow \infty$ we have

$$
\begin{aligned}
& \mathbf{h}_{k}^{\prime}{ }^{\mathrm{H}} \mathbf{W} \mathbf{U}_{k}^{\mathrm{H}} \mathbf{U}_{k} \mathbf{W} \mathbf{h}_{k}^{\prime} \\
& \rightarrow \frac{\mathcal{S}_{\mathbf{V}_{k}^{\mathrm{H}} \mathbf{V}_{k}+\alpha \boldsymbol{\Theta}_{\mathrm{T}}^{-1}}(0)-\alpha \frac{\mathrm{d}}{\mathrm{d} x} \mathcal{S}_{\boldsymbol{\Theta}_{\mathrm{T}}^{1 / 4} \mathbf{V}_{k}^{\mathrm{H}} \mathbf{V}_{k} \boldsymbol{\Theta}_{\mathrm{T}}^{1 / 4}+\alpha \boldsymbol{\Theta}_{\mathrm{T}}^{-1 / 2}}(0)}{\left(1+\mathcal{S}_{\mathbf{V}_{k}^{\mathrm{H}} \mathbf{V}_{k}+\alpha \boldsymbol{\Theta}_{\mathrm{T}}^{-1}}(0)\right)^{2}}
\end{aligned}
$$

Asymptotically, the removal of a single column in the large matrix $\mathbf{H}_{w}^{\prime}$ does not affect the normalized trace in (44) and (49), hence we have

$$
\begin{aligned}
& \mathcal{S}_{\mathbf{V}_{k}^{\mathrm{H}} \mathbf{V}_{k}+\alpha \boldsymbol{\Theta}_{\mathrm{T}}^{-1}}(z)=\mathcal{S}_{\mathbf{H}_{w}^{\prime}{ }^{\mathrm{H}} \mathbf{H}_{w}^{\prime}+\alpha \boldsymbol{\Theta}_{\mathrm{T}}^{-1}}(z)
\end{aligned}
$$

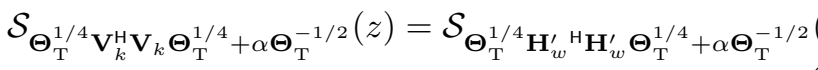

As a consequence, the expression for the asymptotic SINR (39) is user-independent and given by Equation (25).

Furthermore $\mathcal{S}_{\mathbf{H}_{w}^{\prime}{ }^{H} \mathbf{H}_{w}^{\prime}+\alpha \boldsymbol{\Theta}_{\mathrm{T}}^{-1}}(0)$ is the solution of the fixedpoint equation

$$
\mathcal{S}_{\mathbf{H}_{w}^{\prime}{ }^{H} \mathbf{H}_{w}^{\prime}+\alpha \boldsymbol{\Theta}_{\mathrm{T}}^{-1}}(0)=\int \frac{\mu_{\boldsymbol{\Theta}_{\mathrm{T}}}(\nu) \mathrm{d} \nu}{\frac{\alpha}{\nu}+\frac{1}{\beta}\left(1+\mathcal{S}_{\mathbf{H}_{w}^{\prime}{ }^{H} \mathbf{H}_{w}^{\prime}+\alpha \boldsymbol{\Theta}_{\mathrm{T}}^{-1}}(0)\right)^{-1}}
$$

and, from Theorem 1,

$$
\begin{aligned}
& \mathcal{S}_{\boldsymbol{\Theta}_{\mathrm{T}}^{1 / 4} \mathbf{H}_{w}^{\mathrm{H}} \mathbf{H}_{w} \boldsymbol{\Theta}_{\mathrm{T}}^{1 / 4}+\alpha \boldsymbol{\Theta}_{\mathrm{T}}^{-1 / 2}(0)} \\
& =\int\left(\alpha \lambda^{-1 / 2}+\frac{\lambda^{1 / 2}}{\beta(1+\beta \mathcal{T}(0))}\right)^{-1} \mu_{\boldsymbol{\Theta}_{\mathrm{T}}}(\lambda) \mathrm{d} \lambda
\end{aligned}
$$

with $\mathcal{T}(0)$ the unique solution of

$$
\mathcal{T}(0)=\int \frac{\lambda^{1 / 2}}{\beta}\left(\alpha \lambda^{-1 / 2}+\frac{\lambda^{1 / 2}}{\beta(1+\beta \mathcal{T}(0))}\right)^{-1} \mu_{\Theta_{\mathrm{T}}}(\lambda) \mathrm{d} \lambda
$$


Our interest lies however in the derivative of Equation (58), which we compute as

$$
\begin{aligned}
& \frac{\mathrm{d}}{\mathrm{d} x} \mathcal{S}_{\boldsymbol{\Theta}_{\mathrm{T}}^{1 / 4} \mathbf{H}_{w}^{\mathrm{H}} \mathbf{H}_{w} \boldsymbol{\Theta}_{\mathrm{T}}^{1 / 4}+\alpha \boldsymbol{\Theta}_{\mathrm{T}}^{-1 / 2}(0)} \\
& =\int \frac{\lambda^{1 / 2} \frac{\mathrm{d}}{\mathrm{d} x} \mathcal{T}(0)[1+\beta \mathcal{T}(0)]^{-2}+1}{\left(\alpha \lambda^{-1 / 2}+\lambda^{1 / 2}(\beta[1+\beta \mathcal{T}(0)])^{-1}\right)^{2}} \mu_{\boldsymbol{\Theta}_{\mathrm{T}}}(\lambda) \mathrm{d} \lambda
\end{aligned}
$$

where $\mathcal{T}(0)$ verifies Equation (59) and $\frac{\mathrm{d}}{\mathrm{d} x} \mathcal{T}(0)$ is the only solution of the fixed-point equation

$$
\frac{\mathrm{d}}{\mathrm{d} x} \mathcal{T}(0)=\int \frac{\lambda \frac{\mathrm{d}}{\mathrm{d} x} \mathcal{T}(0)(\beta[1+\beta \mathcal{T}(0)])^{-2}+\frac{\lambda^{1 / 2}}{\beta}}{\left(\frac{\alpha}{\lambda^{1 / 2}}+\lambda^{1 / 2}(\beta[1+\beta \mathcal{T}(0)])^{-1}\right)^{2}} \mu_{\Theta_{\mathrm{T}}}(\lambda) \mathrm{d} \lambda
$$

Finally, the corresponding per-user rate is expressed directly from the SINR in Equation (25) and reads

$$
\mathcal{R}_{\mathrm{rzf}, k} \rightarrow \log (1+\gamma)
$$

Remark 2: We remind that, in this regularization scenario, the normalization parameter $\alpha$ is chosen such that (31) is satisfied. The authors in [14] chose a slightly different approach to the R-ZF. The precoding matrix reads

$$
\mathbf{G}_{\mathrm{rzf}}=\xi(\alpha)\left(\mathbf{H}^{\mathrm{H}} \mathbf{H}+M \alpha \mathbf{I}_{M}\right)^{-1} \mathbf{H}^{\mathrm{H}}
$$

where $\xi(\alpha)$ is used to normalize the transmit power (8) and the free parameter $\alpha$ is adapted to maximize the sum rate. With a similar derivation as previously the asymptotic SINR $\gamma(\alpha)$ for a given $\alpha$ is the same as in (25) except for the term $(1+$ $\left.\mathcal{S}_{\mathbf{H}_{w}^{\mathrm{H}} \mathbf{H}_{w}+\alpha \boldsymbol{\Theta}_{\mathrm{T}}^{-1}}(0)\right)^{2}$ which becomes $\left(1+\mathcal{S}_{\mathbf{H}_{w}^{\mathrm{H}} \mathbf{H}_{w}+\alpha \boldsymbol{\Theta}_{\mathrm{T}}^{-1}}(0)\right)^{2}$. $\Psi(\alpha)$ with

$$
\Psi(\alpha)=\mathcal{S}_{\mathbf{H}^{\prime H} \mathbf{H}^{\prime}}(-\alpha)-\alpha \frac{\mathrm{d}}{\mathrm{d} x} \mathcal{S}_{\mathbf{H}^{\prime H} \mathbf{H}^{\prime}}(-\alpha)
$$

and the asymptotic achievable per-user rate is

$$
\mathcal{R}_{\mathrm{rzf}, k}=\sup _{\alpha} \log (1+\gamma(\alpha))
$$

The complete derivation of this result is provided in [20].

\section{Simulation and Results}

In this section we apply the asymptotic results obtained in the previous sections to a MIMO-BC with $K$ users and an $M$ antenna UCA at the transmitter; the asymptotic eigenvalues of the transmit correlation matrix are therefore directly given by (11). Also, for small $K$, comparison is made between Monte-Carlo simulations of the ergodic sum rate and the asymptotic formulas applied for the discrete density function $\mu_{\Theta_{\mathrm{T}}}(\lambda)=\frac{1}{M} \sum_{m=1}^{M} \delta\left(\lambda-\lambda_{m}\right)$, with $\left\{\lambda_{m}\right\}$ the eigenvalues of $\Theta_{\mathrm{T}}$. Note that the SINR (19) and (25) are easily computed by solving the various fixed-point equations recursively. ${ }^{2}$

Figures 2 and 3 show the sum rates of ZF and R-ZF, respectively, for $M / K=1.5,1 / \sigma^{2}=10 \mathrm{~dB}$ and different

\footnotetext{
${ }^{2}$ the authors do not however claim that the recursive processes always converge. Still, as far as this section is concerned, all fixed-point equations converged.
}

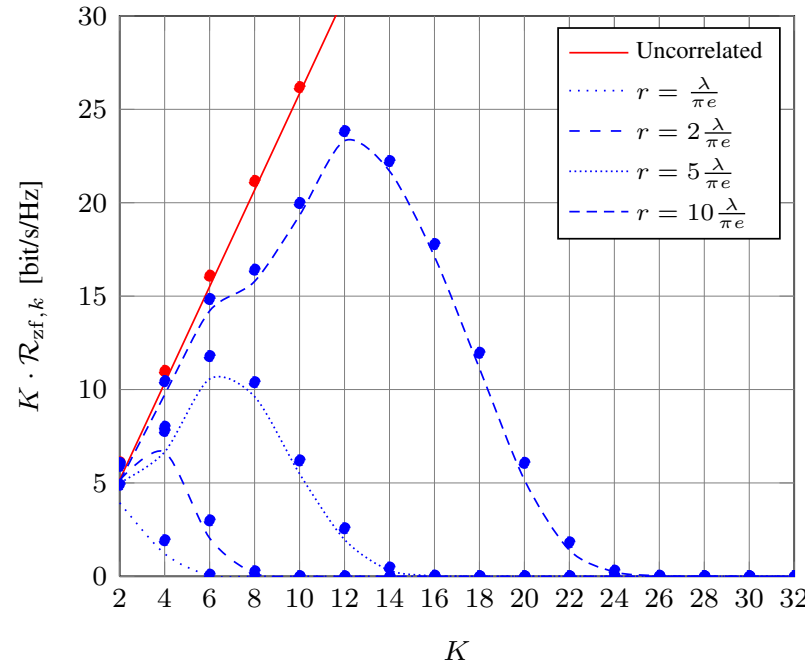

Fig. 2. ZF, UCA, Ergodic MIMO sum rate $K \cdot \mathcal{R}_{\mathrm{zf}, k}$ for different $r / \lambda$, $\beta=1.5, \frac{1}{\sigma^{2}}=10 \mathrm{~dB}$, numerical simulations indicated by circle marks

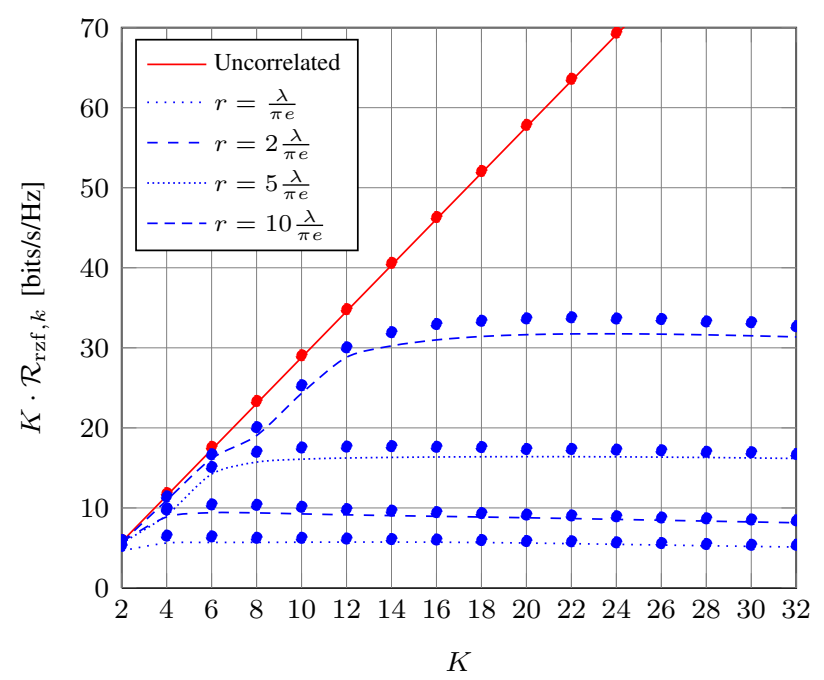

Fig. 3. R-ZF, UCA, Ergodic MIMO sum rate $K \cdot \mathcal{R}_{\mathrm{zf}, k}$ for different $r / \lambda$, $\beta=1.5, \frac{1}{\sigma^{2}}=10 \mathrm{~dB}$, numerical simulations indicated by circle marks

ratios $r / \lambda$ between the array radius and the signal wavelength. It can be observed that the sum rate for $\mathrm{ZF}$ goes rapidly to zero for large $K$, because the channel matrix becomes singular when the transmit antennas are strongly correlated. We observe that the maximum simulated sum rate occurs for $K$ slightly larger than the number of degrees of freedom $\lceil\pi e r / \lambda\rceil$. On the other hand the simulated sum rate of R-ZF is almost constant for large $K$, where it converges to the theoretical asymptotic sum rate. Moreover, the asymptotic sum rate is higher than the maximum simulated sum rate of $\mathrm{ZF}$.

Surprisingly, we observe that, even for small $K$, the asymptotic approximation closely matches the simulated points, both for ZF and R-ZF. This can be explained by the fact that instantaneous sum rates have a small variance around the ergodic sum rate, especially for $\beta>1$. However, we 
emphasize that our asymptotic expressions are only valid for $K \rightarrow \infty$ and cannot be relied on for small $K$. In particular, in the $\mathrm{ZF}$ scenario with $\beta=1$, when $K$ is small, simulations show that the asymptotic approximation is inaccurate. This is thoroughly discussed in [20].

\section{CONCLUSION}

This paper provides expressions for the sum rate of the MIMO-BC with correlated antennas for ZF and R-ZF precoding when the number of users grows large. The analytical formulas are particularly appealing since they are based on tractable and numerically solvable fixed-point equations. The results are applied to the problem of dense antenna packing on volume-limited transmitters. In this case, the asymptotic formulas are verified and, when applied to small numbers of users, are shown to be generally good approximations to the actual sum rate under linear precoding.

\section{ACKNOWLEDGMENT}

The authors would like to thank Jack Silverstein for his valuable help with Theorem 1 . This work was partially supported by the European Commission in the framework of the FP7 Network of Excellence in Wireless COMmunications $\mathrm{NEWCOM++.}$

\section{REFERENCES}

[1] G. J. Forschini and M. J. Gans, "On Limits of Wireless Communications in a Fading Environment when Using Multiple Antennas", Wireless Personal Communications, vol. 6, no. 3, pp. 311-335, 1998

[2] I.E. Telatar, "Capacity of multi-antenna Gaussian channels," Tech. Rep., ATT Bell Labs, 1995

[3] T.S. Pollock, T.D. Abhayapala, and R.A. Kennedy, "Antenna saturation effects on dense array MIMO capacity," IEEE Intl. Conf. on Commun., ICC'03, Anchorage, Alaska, May. 11-15 2003, pp. 2301-2305.

[4] P. Viswanath, D. Tse, "Sum capacity of the vector Gaussian broadcast channel and uplink-downlink duality," vol. 49, no. 8, pp. 1912-1921, 2003

[5] G. Caire and S. Shamai, "On the achievable throughput of a multiantenna Gaussian broadcast channel," IEEE Trans. on Information Theory, vol. 49, no. 7, pp. 1691-1706, 2003

[6] A.M. Tulino, A. Lozano, and S. Verdú, "Impact of correlation on the capacity of multi-antenna channels," IEEE Trans. on Information Theory, vol. 51, no. 7, pp. 2491-2509, 2005

[7] P. Dent, G. E. Bottomley and T. Croft, "Jakes fading model revisited," Electonics Letters, vol. 29, no. 13, June 1993

[8] J. W. Silverstein and Z.D Bai, "On the empirical distribution of eigenvalues of a class of large dimensional random matrices," Journal of Multivariate Analysis, vol. 54, issue 2, pp. 175-192, 1995

[9] A. M. Tulino, S. Verdú, "Random Matrix Theory and Wireless Communications," Now Publishers, vol. 1, issue 1, 2004

[10] Z. D. Bai and J. W. Silverstein, "No eigenvalue outside the support of the limiting spectral distribution of large dimensional sample covariance matrices," Ann. Probab. vol. 26, no. 1, pp. 316-345, 1998

[11] M. Debbah and R. Müller, "MIMO Channel Modeling and the Principle of Maximum Entropy," IEEE Transactions on Information Theory, vol. 51, no. 5, May 2005

[12] E. Wigner, "Random Matrices in Physics," SIAM Review, vol. 9, no. 1, January, 1967.

[13] B.M. Hochwald and S. Vishwanath, "Space-Time Multiple Access: Linear Growth in the Sum Rate," in Proc. 40th Allerton Conf. Comput., Commun., Control, vol. 40, no. 1, pp. 387-396, 2002

[14] C. Peel, B. Hochwald and A. Swindlehurst, "A Vector-Perturbation Technique for Near-Capacity Multiantenna Multiuser Communication - Part I: Channel Inversion and Regularization," IEEE Trans. on Communications, vol. 53, no. 1, pp. 195-202, Jan. 2005
[15] C. Peel, B. Hochwald and A. Swindlehurst, "A Vector-Perturbation Technique for Near-Capacity Multiantenna Multiuser Communication - Part II: Perturbation,' IEEE Trans. on Communications, vol. 53, no. 3, pp. 537-544, Mar. 2005

[16] T. Muharemovic, A. Sabharwal and B. Aazhang, "Antenna Packing in Low-Power Systems: Communication Limits and Array Design," IEEE Trans. on Information Theory, vol. 54, no. 1, pp. 429-440, 2008

[17] A.S.Y. Poon, R.W. Brodersen and D.N.C. Tse, "Degrees of Freedom in Multiple-Antenna Channels: A Signal Space Approach," IEEE Trans. on Information Theory, vol. 51, no. 2, pp. 523-536, 2005

[18] D. Gesbert, T. Ekman and N. Christophersen, "Capacity Limits of Dense Palm-Sized MIMO Arrays," Globecom'02, Taipei, Taiwan 2002

[19] C. Chuah, D.N.C. Tse, J.M. Kahn, R.A.V. Valenzuela, "Capacity Scaling in MIMO Wireless Systems Under Correlated Fading," IEEE Trans. on Information Theory, vol. 48, no. 3, pp. 637-650, 2002

[20] R. Couillet, S. Wagner, M. Debbah and A. Silva, "Asymptotic Analysis of Correlated Multi-Antenna Systems", submitted to IEEE Trans. on Information Theory 